\title{
INFLUENCE OF ACOUSTICALLY EXCITED AIRFLOWS ON A PLANAR AIRBLAST PREFILMER
}

\author{
Thomas Christou \\ Karlsruhe Institute of Technology \\ Engler-Bunte-Institute \\ Karlsruhe, Germany
}

\author{
Björn Stelzner \\ Karlsruhe Institute of Technology \\ Engler-Bunte-Institute \\ Karlsruhe, Germany
}

\author{
Nikolaos Zarzalis \\ Karlsruhe Institute of Technology \\ Engler-Bunte-Institute \\ Karlsruhe, Germany
}

\section{ABSTRACT}

In order to meet the higher requirements for clean combustion technology in aircraft engine applications and thus reduce harmful emissions, especially nitrogen oxide emissions, the major jet engine manufacturers are developing lean premixed prevaporized (LPP) combustors that operate at very high pressure. In this context, thermoacoustic instabilities may occur within the combustion chamber. The unsteady heat released by the flame generates pressure waves, which are coupled to the inlet air velocity by a feedback loop. This loop amplifies the instabilities of the inlet air velocity, which in turn influences the atomization process.

Since the atomization process at the airblast atomizers of most jet engine combustors determines critical operating characteristics such as air-to-fuel ratio (AFR), flame stability, or $N O_{x}$ emissions, predicting the performance of this process under unsteady conditions has a significant value.

The present experimental study focuses on the influence of oscillating airflows on the spray characteristics at the airblast atomization process. The experimental setup was based on a two-dimensional prefilmer where a water film flow was introduced on one surface. The airflow was excited by a siren, whereby an excitation frequency near $94 \mathrm{~Hz}$ was investigated. The airflow oscillation under this excitation frequency was characterized using a Constant Temperature Anemometer (CTA), while the generated spray was investigated with a Phase Doppler Anemometry (PDA) system.

The spray was investigated in a variety of positions along the radial axis, providing spatial information, apart from temporal. The characterization of the spray via PDA includes a two-component droplet velocity detection and diameter measurement, while the spray mass flux for each measured position was also calculated. The acquired data were phase averaged via an in-house developed processing algorithm, while through a statistical analysis the confidence intervals of the calculations were included. The excitation frequency strongly influenced all spray characteristics, namely, the Sauter Mean Diameter (SMD), the droplet velocities, the mass flux, as well as the local air-to-liquid ratio (ALR). Depending on the phase angle, the size distribution of the spray changes, explaining the observed oscillating behavior of the spray characteristics.

Keywords: Atomization and sprays, Thermoacoustics, Jet engines

\section{NOMENCLATURE}

A

d

$\mathrm{D}_{63.2 \%}$

$\dot{\mathrm{m}}$

$\dot{\mathrm{m}}^{\prime \prime}$

$\mathrm{N}$

Q

q

$\mathrm{t}$

u

$\mathrm{X}$

$\mathrm{Y}$

Z

\section{Greek}

$\Delta(\cdot)$

$\rho$

$\varphi$

Effective detection area

Droplet diameter

Rosin-Rammler parameter

Mass flow rate

Mass flux

Number of droplets

Liquid volume fraction

Rosin-Rammler parameter

Time

Velocity

Horizontal coordinate in spray

Depth coordinate in spray

Vertical coordinate in spray

$\left[\mathrm{mm}^{2}\right]$

$[\mu \mathrm{m}]$

$[\mu \mathrm{m}]$

$[\mathrm{kg} / \mathrm{s}]$

$\left[\mathrm{kg} / \mathrm{m}^{2} \mathrm{~s}\right]$

[-]

$[-]$

[-]

[ms]

$[\mathrm{m} / \mathrm{s}]$

[mm]

[mm]

[mm]

\section{Acronyms}

ALR

GALR

LPP

NACA

PDA

SMD
Difference

Density

Phase Angle

$[-]$ $\left[\mathrm{kg} / \mathrm{m}^{3}\right]$ $\left[{ }^{\circ}\right]$

Air-to-Liquid Ratio

Global Air-to-Liquid Ratio

Lean Premixed Prevaporized

National Advisory Committee for Aeronautics

Phase Doppler Anemometry

Sauter Mean Diameter 


\section{INTRODUCTION}

The concept of lean premixed prevaporized (LPP) combustion has attracted the interest of numerous jet engine manufacturers over the past years due to its low $\mathrm{NO}_{x}$ emissions [1]. However, a combustion chamber operating at high pressures with a preheated air supply in the context of LPP is prone to naturally grown instabilities with destructive potential [2-4]. These thermoacoustic instabilities occur when the heat release is unstable and the produced pressure waves in the chamber are linked with the inlet air velocity, which further enhances the instability of the released heat.

The performance of the airblast atomizers employed on most combustion chambers of modern jet engines affects the flame stability, soot formation, $\mathrm{NO}_{\mathrm{x}}$ emissions, as well as the local air-to-fuel ratio (AFR), which is the mass flow of air over the fuel mass flow on each location of the spray. It is therefore apparent, that an unsteady heat release influencing the atomization process can further affect major operating conditions on the engine. The demand to predict the airblast atomization performance has been evident over the last decades.

Experimental investigations by Eckstein et al. [5] have given insights into the airflow and the spray characteristics under acoustic forcing at low excitation frequencies in the range of 50 to $150 \mathrm{~Hz}$, showing that the measured Sauter Mean Diameter (SMD) under oscillating airflow matches the steady atomization results. Other measurements by Müller et al. [6] on a model planar atomizer showed that the droplet diameter responds almost proportionally to the air velocity fluctuations for excitation frequencies below $350 \mathrm{~Hz}$. By taking the results from Müller a step further, Chaussonnet et al. [7] used models to predict the volume probability density function and match the prediction to the experimental data obtained by means of shadowgraphy. In a more recent study, Su et al. [8] attempted to incorporate experimental data obtained via Phase Doppler Anemometry (PDA) in empirical correlations for SMD predictions, in order to investigate the phase portraits of SMD versus gas velocity in a CFD simulation of a three-injector leanburn facility.

In the present experimental work, the droplet characteristics were measured using PDA, in a setup where a pulsation device imposed a modulation in the airflow. The measured airflow oscillations via hot wire anemometry along with all measured spray characteristics were post-processed in order to discuss their phase-averaged behavior for one period. All experiments were conducted in a variety of radial positions, providing spatial information about the periodic behaviors. Apart from the droplet velocity in two directions and the droplet diameter given by PDA, insights on the spray angle and its variation in time were given. To quantify the periodic variation of the size distribution of the spray, a Rosin-Rammler model was applied to the phaseaveraged data. The periodic oscillation of the mass flux was calculated, after evaluating the calculation algorithm in nonforced conditions experiments. By combining the information from the measured airflow velocity and the calculated mass flux, the periodic fluctuation of the local air-to-liquid ratio (ALR) was calculated, showing a relatively high oscillation amplitude.

\section{EXPERIMENT \\ Experimental facility}

The schematic of the setup in which the measurements were conducted is shown in Figure 1. A compressor supplies air into the system, while the airflow is split into two sections; part of the airflow passes through a siren and is subject to an imposed acoustic forcing, while the rest of the airflow is bypassed through an air plenum. The two streams mix and enter coaxially a $1.5 \mathrm{~m}$ long tube, at the end of which an airblast prefilmer is positioned. Water is supplied directly inside the prefilmer from a vessel maintained under constant pressure using a 200 bar nitrogen bottle. The water flow rate is controlled by a set of needle valves and a mass flow meter. Finally, the sprayed liquid is collected in a tank, from where the air is sucked by a vacuum pump, ensuring that it does not cause a recirculation in the spray region affecting the measurement.

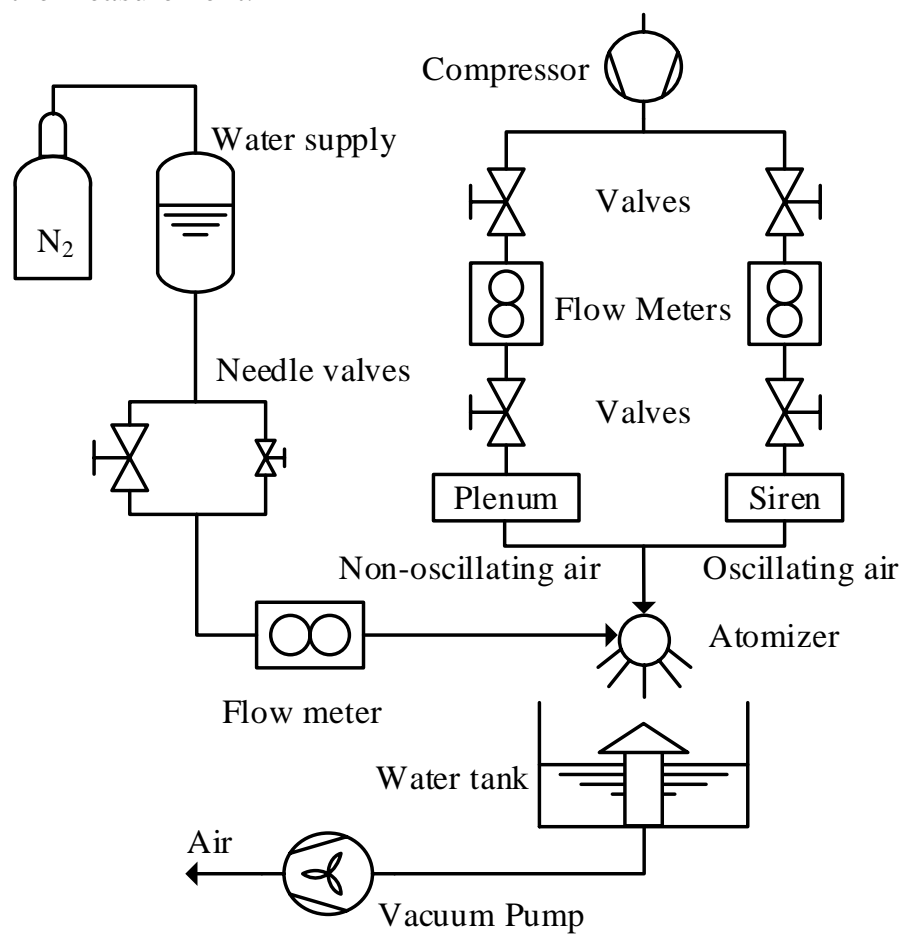

FIGURE 1: TEST RIG SCHEMATIC.

The atomizer designed for this work is a planar model of a prefilming airblast nozzle, similar to the design proposed by Müller et al. [6]. The prefilmer has a profile of a standardized NACA airfoil with a chord length of $73 \mathrm{~mm}$ and a width of $70 \mathrm{~mm}$. It is made of stainless steel with an atomization edge thickness of about $200 \mu \mathrm{m}$. The liquid is entering the inside of the prefilmer into a cavity, symmetrically from both sides through the sidewalls. From this cavity, the liquid emerges on the surface through 40 holes of diameter $0.5 \mathrm{~mm}$ each, creating a uniform thin film with a width of approximately $40 \mathrm{~mm}$ that flows to the edge where it is being atomized. This planar airblast prefilmer is illustrated in a cut section in Figure 2. On the same sketch, the measurement positions are displayed exaggerated, along with the positive axes directions and their origins. 


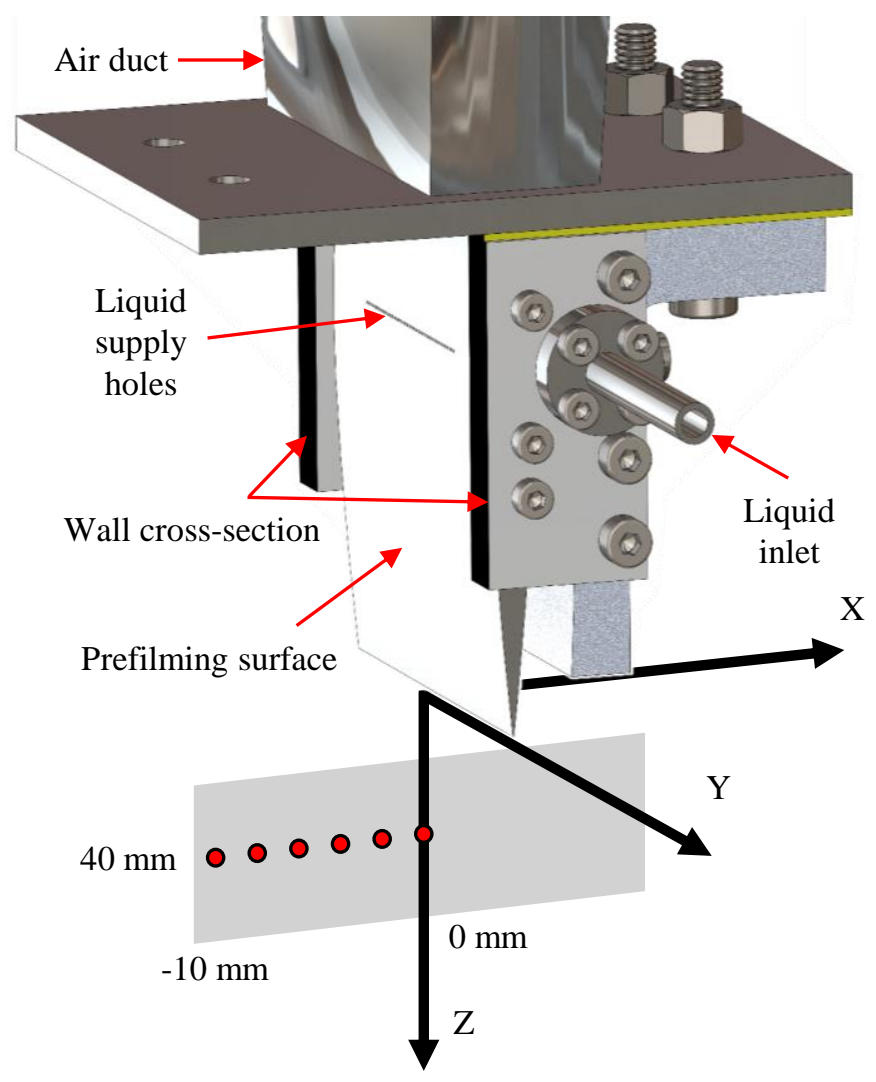

FIGURE 2: PLANAR PREFILMING AIRBLAST ATOMIZER AND MEASUREMENT POSITIONS (NOT TO SCALE).

The siren, designed and manufactured at the Technical University of Munich [5], consists of a rotating disc attached to a motor on the inside of a stationary housing. During the rotation, when the openings of the disc coincide with the openings of the housing shell, the air is allowed to pass. The rotational speed of the motor, and therefore the excitation frequency, is controlled via computer software.

The part of the airflow that does not go through the siren is bypassed through the air plenum designed to minimize the pressure losses. The airflow exiting the plenum through several holes surrounds the airflow that has emerged from the siren; therefore, the two flows mix coaxially inside the tube upstream of the prefilmer.

\section{Measurement techniques}

The investigation of the generated airflow under the acoustic forcing was done using hot wire Constant Temperature Anemometry (CTA). A two-wire probe was employed, with tungsten platinum-coated wires of $3.8 \mu \mathrm{m}$ diameter and $1.27 \mathrm{~mm}$ length each. The probe was mounted on a translation stage enabling accurate movement inside the flow field, so the airflow was measured on the positions marked in Figure 2. A high sampling rate of $20,000 \mathrm{~Hz}$ was selected for this application in order to safely identify the oscillations in the $100 \mathrm{~Hz}$ order of magnitude, collecting approximately 262,000 samples for each position.
To characterize the spray, a commercial Phase Doppler Anemometry (PDA) system was employed. The PDA setup involved a three-detector receiving optic at a scattering angle of $30^{\circ}$, utilizing the first-order refraction mode. A diode-pumped solid-state laser was used, providing laser beams in the wavelengths of $532 \mathrm{~nm}$ (green) and $561 \mathrm{~nm}$ (yellow). The vertical component of the droplet velocity (parallel to the Z-axis) and the droplet diameter measurement was accomplished with the green line, while for the measurement of the horizontal component of the droplet velocity (parallel to the $\mathrm{X}$-axis) the yellow line was used. Considering the wavelength of the laser beams, the beam spacing, and their intersection angle, this setup configuration generates a measurement volume with a diameter of approximately $154 \mu \mathrm{m}$ and a length of approximately $2.7 \mathrm{~mm}$, which enables measurement for droplet diameters up to $300 \mu \mathrm{m}$. A data rate of up to $10 \mathrm{kHz}$ was achieved when measuring in dense spray regions, with an average validation rate of approximately $60 \%$. In the experiments, 100,000 droplet samples were acquired for each of the measured positions shown in Figure 2 and then phase-averaged.

The positions selected for the PDA measurements, which match the measurement positions of the airflow characterization via hot wire anemometry, are shown in Figure 2. A downstream distance of $40 \mathrm{~mm}$ in the Z-axis was selected in order to scan the $\mathrm{X}$-axis in six different positions $(-10 \mathrm{~mm}$ to $0 \mathrm{~mm})$ in this radial direction. Preliminary measurements in non-forced conditions had shown that the spray is uniform along the $\mathrm{Y}$-axis in this downstream distance; therefore, the six positions lie in the center of the prefilmer at $\mathrm{Y}=0 \mathrm{~mm}$.

Since low-frequency instabilities between 50 and $150 \mathrm{~Hz}$ are of particular interest in aero-engine combustor applications [5], a variety of frequencies in the range of 90 to $150 \mathrm{~Hz}$ was originally investigated. For this study, an excitation frequency at $93.5 \mathrm{~Hz}$ was selected as one of the cases with negligible influence of harmonics. The water mass flow rate was maintained constant throughout the experiments; therefore, the global ALR was fluctuating around an average of 26.

On the displayed results of the air and spray characteristics, a confidence interval was added to provide a statistical value of the data. A confidence level of $95 \%$ was selected in the calculation using either the bootstrap resampling method with 10,000 bootstrap samples for each analyzed variable or the Student's t-test $[9,10]$.

\section{RESULTS AND DISCUSSION Unsteady airflow velocity}

Before measuring the spray characteristics, the velocity of the airflow under the influence of forcing induced by the siren at 93.5 Hz was measured in the six positions shown in Figure 2. By phase-averaging the airflow total velocity signal, the oscillation in the flow distinctively shows, as observed from Figure 3. To enable comparability between the air velocity and the spray characteristics in each of the six selected positions, since they were measured independently using different techniques, the phase-averaged air velocity signal was shifted in order to match the phase angle of the phase-averaged droplet velocity of 
droplets with a diameter less than $10 \mu \mathrm{m}$. This way, the assumption that the smallest droplets of the spray do not have any phase shift was made, and thus the rest of the droplet characteristics depicted in this study correspond to the air velocity phase angle shown in Figure 3. The total air velocity fluctuates with an amplitude of approximately $10 \mathrm{~m} / \mathrm{s}$, while it does not significantly vary in the selected $10 \mathrm{~mm}$ interval along the $\mathrm{X}$-axis.

\begin{tabular}{|c|c|c|c|c|c|}
\hline$\Phi$ & $\begin{array}{l}X=-10 \mathrm{~mm} \\
X=-8 \mathrm{~mm}\end{array}$ & $\frac{\bar{\Phi}}{\overline{\underline{1}}}$ & $\begin{array}{l}X=-6 \mathrm{~mm} \\
X=-4 \mathrm{~mm}\end{array}$ & $\frac{\Phi}{\Phi}$ & $\begin{array}{l}X=-2 \mathrm{~mm} \\
X=0 \mathrm{~mm}\end{array}$ \\
\hline
\end{tabular}

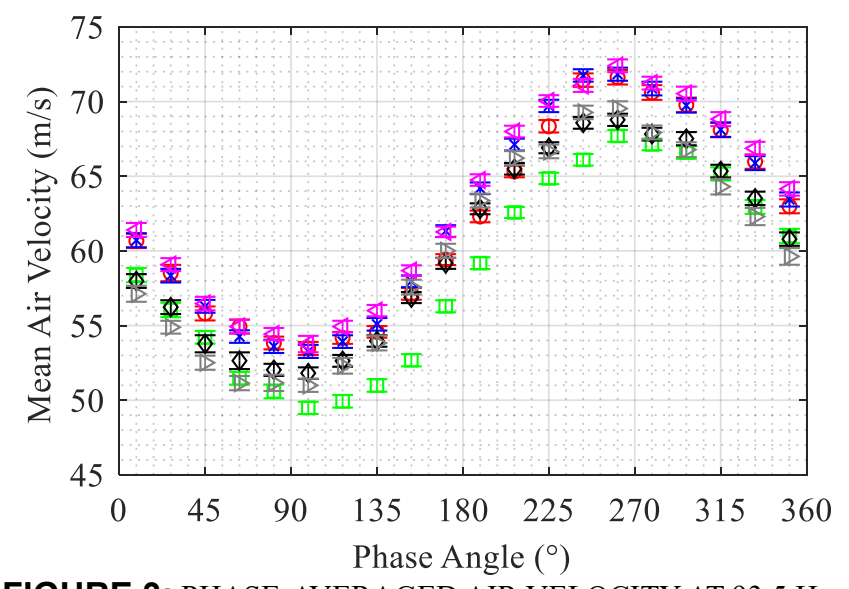

FIGURE 3: PHASE-AVERAGED AIR VELOCITY AT $93.5 \mathrm{~Hz}$.

\section{Basic spray characteristics}

To properly analyze the data measured under the acoustic forcing, they were firstly phase-averaged for one period of the phenomenon. This means that for each measurement position, the period was divided into twenty subsections and, therefore, the periodic oscillation is observed.

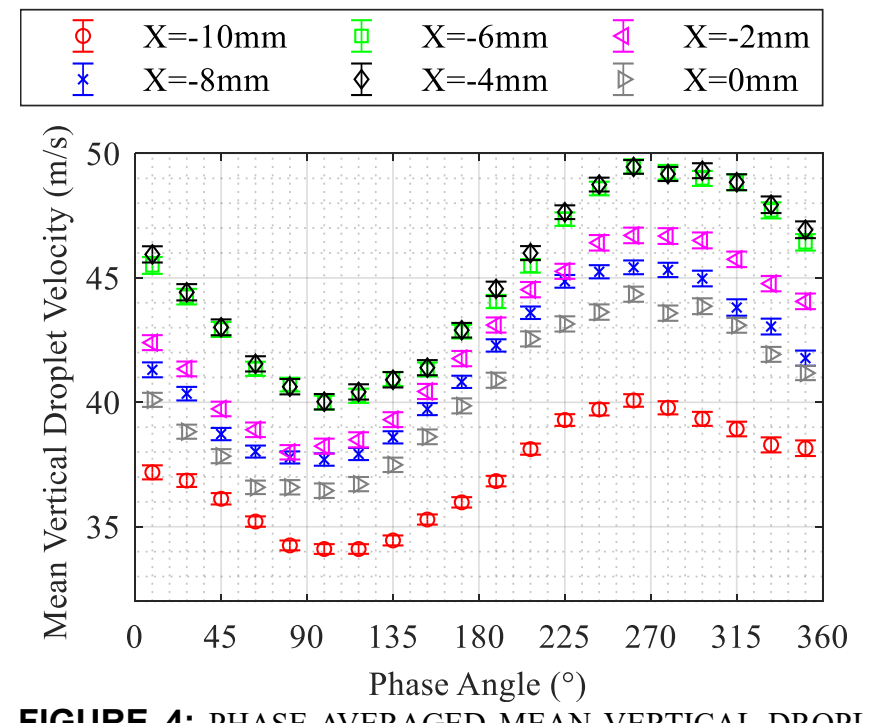

FIGURE 4: PHASE-AVERAGED MEAN VERTICAL DROPLET VELOCITY AT $93.5 \mathrm{~Hz}$.
The mean vertical droplet velocity, measured from the green line (532 nm wavelength) of the PDA system, is illustrated in Figure 4 , where a clear periodic oscillation over time is observed for all different measured positions. The average from approximately 5,000 samples per sub section is quite reliable, since the $95 \%$ confidence interval calculated with the Student's t-test is so narrow. Along the $\mathrm{X}$-axis, a local maximum in the mean vertical component of the droplet velocity appears approximately 4 to $6 \mathrm{~mm}$ away from the centerline, where the velocity of the airflow was relatively constant as observed in Figure 3.

Since the measurement technique employed for this research involved a 2D PDA system, by utilizing the yellow line (561 nm wavelength) of the laser, the velocity component parallel to the $\mathrm{X}$-axis was also measured. As expected, this horizontal velocity component is increasing in absolute value moving radially from the centerline, as seen in Figure 5. The oscillation in time is also evident, with a phase matching to the vertical velocity component. The amplitude of the oscillation in the horizontal velocity is increasing with increasing absolute mean value moving from $X=0 \mathrm{~mm}$ to $X=-10 \mathrm{~mm}$.

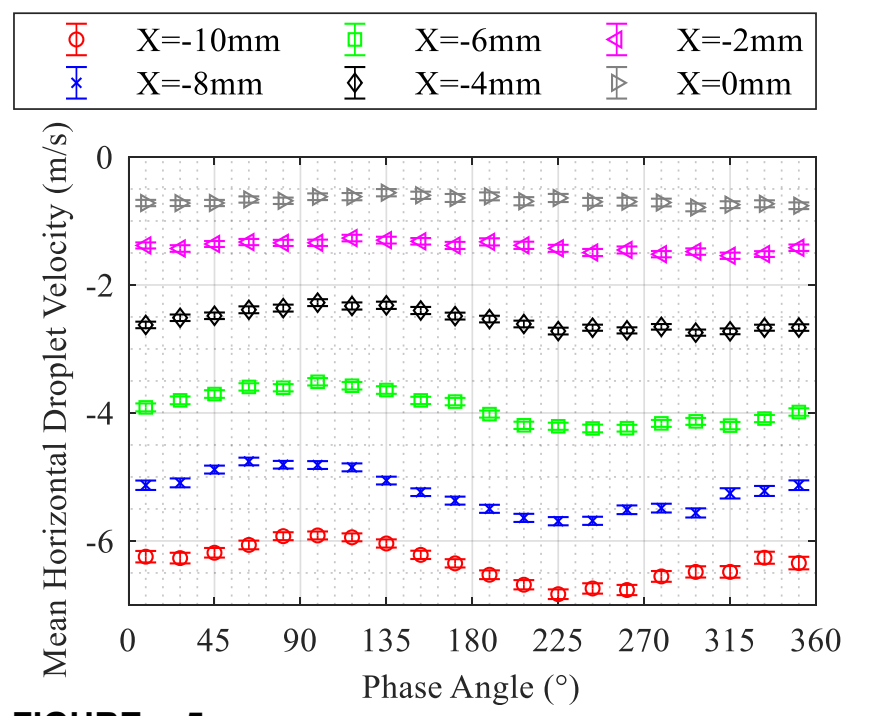

FIGURE 5: PHASE-AVERAGED MEAN HORIZONTAL DROPLET VELOCITY AT $93.5 \mathrm{~Hz}$.

This leads to a variation in the velocity angle (i.e. spray halfangle) in space but not in time, as shown in Figure 6. This angle (relative to the $\mathrm{Z}$-axis on the $\mathrm{XZ}$ plane) increases in absolute value at the extremes of the measured interval reaching values of $10^{\circ}$, implying a spray angle of approximately $20^{\circ}$ at a radial distance of $\pm 10 \mathrm{~mm}$ on the X-axis. On the other hand, no significant fluctuation is noticed during the period of the forcing, since the oscillations of the two velocity components cancel each other. This means that an external observer, locked in one position of the spray with an Eulerian approach, would see the droplets always arriving at the same angle, even though their absolute velocity is oscillating in time due to the excitation frequency imposed in the system. 


\begin{tabular}{|llllll|}
\hline$\Phi$ & $X=-10 m m$ & $\Phi$ & $X=-6 m m$ & $\Phi$ & $X=-2 m m$ \\
平 & $X=-8 m m$ & $\overline{\underline{1}}$ & $X=-4 m m$ & $\Phi$ & $X=0 m m$ \\
\hline
\end{tabular}

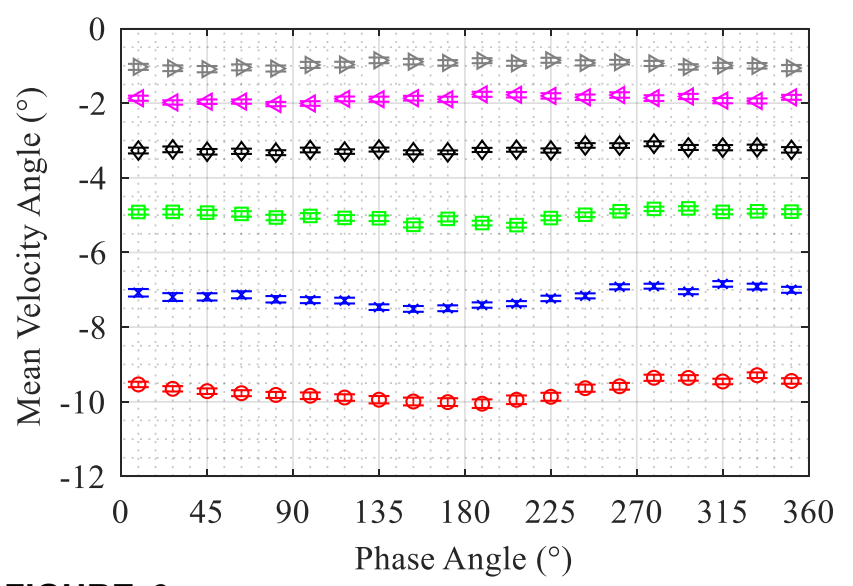

FIGURE 6: PHASE-AVERAGED MEAN DROPLET VELOCITY ANGLE AT $93.5 \mathrm{~Hz}$.

As already mentioned in the description of the PDA setup, the droplet diameter was measured as well. In a similar way to the velocity, it is possible to note how the droplet diameter is changing during time because of the imposed forcing, by observing the Sauter Mean Diameter of the spray. For each of the six measurement positions, the phase-averaged SMD is displayed in Figure 7. The SMD undergoes a clear oscillation during the period, varying with even a difference of $40 \mu \mathrm{m}$. The SMD is smaller when the velocity is higher, which is expected since small droplets tend to move faster due to their inertia [11]; therefore, a spray that consists of smaller droplets has a higher average velocity. A slight drop in the SMD is noticed from the centerline $(\mathrm{X}=0 \mathrm{~mm})$ to the extremes of the measured interval.

\begin{tabular}{|c|c|c|c|c|c|}
\hline$\frac{\Phi}{\bar{x}}$ & $\begin{array}{l}X=-10 \mathrm{~mm} \\
X=-8 \mathrm{~mm}\end{array}$ & $\bar{\Phi}$ & $\begin{array}{l}X=-6 \mathrm{~mm} \\
X=-4 \mathrm{~mm}\end{array}$ & $\frac{\Phi}{\Phi}$ & $\begin{array}{l}X=-2 \mathrm{~mm} \\
X=0 \mathrm{~mm}\end{array}$ \\
\hline
\end{tabular}

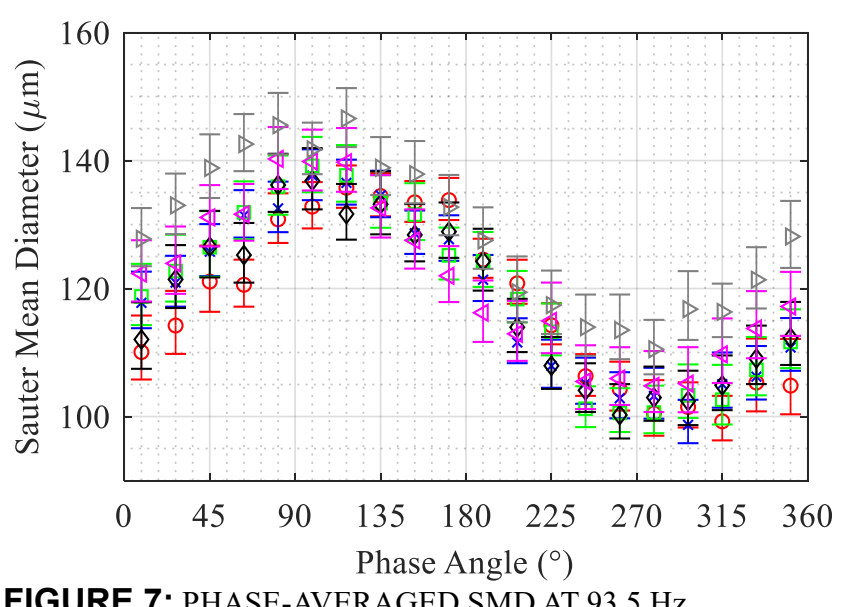

FIGURE 7: PHASE-AVERAGED SMD AT $93.5 \mathrm{~Hz}$.
As established from Figure 4 and Figure 7, the droplet velocity is higher when the spray consists of smaller droplets leading to a lower SMD. This is also apparent in the size-velocity correlation of the spray at each instant. Figure 8 shows this correlation for the position at $\mathrm{X}=0 \mathrm{~mm}$ and how it changes along the period, where the inverse proportionality of the velocity to the diameter is clear. The confidence levels for the presented fit on the droplet data are also displayed for each case.

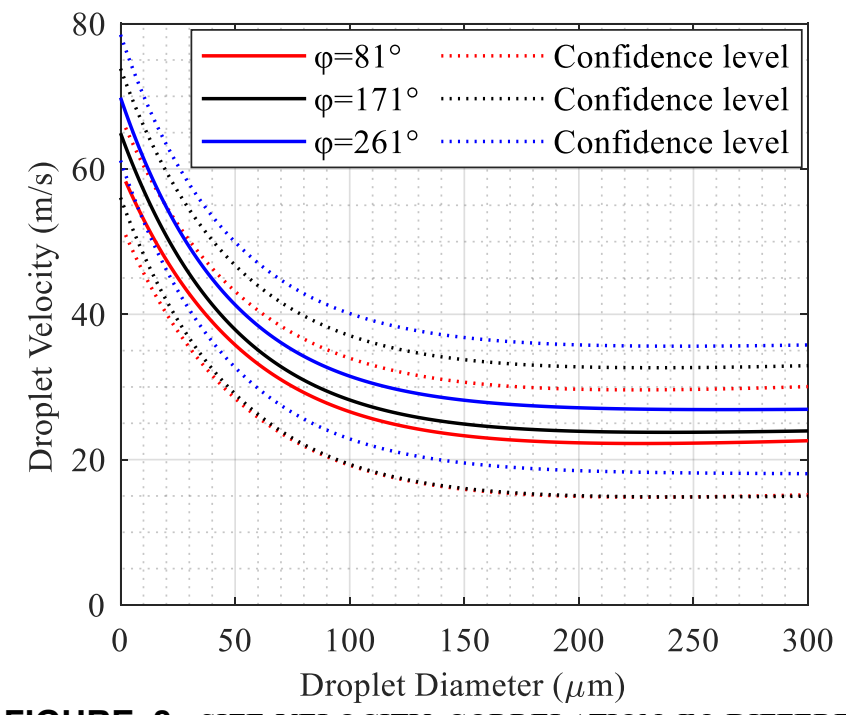

FIGURE 8: SIZE-VELOCITY CORRELATION IN DIFFERENT PHASE ANGLES OF A PERIOD AT $93.5 \mathrm{~Hz}(\mathrm{X}=0 \mathrm{~mm})$.

The strong fluctuation of the SMD of the spray over time can be justified by a change in the diameter distribution along the period. In Figure 9, the droplet diameter distribution is shown as a probability density function for two instances of the period, with a phase difference of $180^{\circ}$, near the maximum and minimum of the SMD fluctuation at the centerline $(X=0 \mathrm{~mm})$. The difference in the shape of the distributions is clear; at $81^{\circ}$, the peak is shorter and there are more droplets with high diameters, hence the higher SMD.

In order to quantify the particle size distribution, various models have been proposed. The most extensively used is the one developed by Rosin and Rammler [12]. The Rosin-Rammler distribution is expressed as:

$$
Q=1-e^{-\left(d / D_{63.2 \%}\right)^{q}}
$$

where $\mathrm{Q}$ is the percentage of the total liquid volume contained in droplets with a diameter lower than $\mathrm{d}$. The parameter $\mathrm{D}_{63.2 \%}$ is the diameter below which $63.2 \%$ of the total liquid volume is contained, while $\mathrm{q}$ is a parameter denoting the spread of the distribution and usually lies between 1.5 and 4 [13]. The application of the Rosin-Rammler equation is shown in an example in Figure 10. The fit on the data, either on the volume fraction or the cumulative volume fraction on the right axis, is reasonably good. 


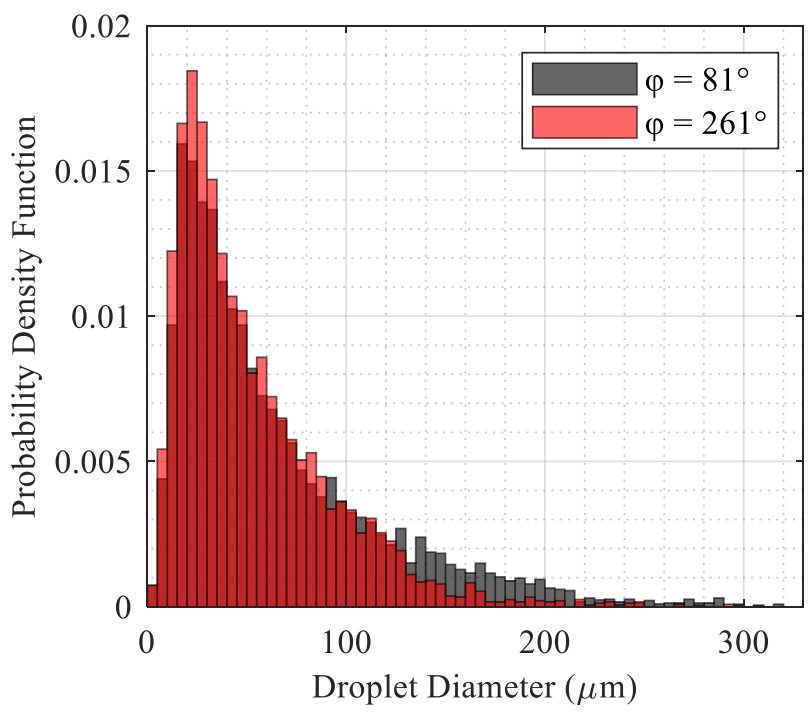

FIGURE 9: PROBABILITY DENSITY FUNCTION OF THE DROPLET DIAMETER AT $\mathrm{X}=0 \mathrm{~mm}$ FOR TWO INSTANCES OF THE PERIOD.
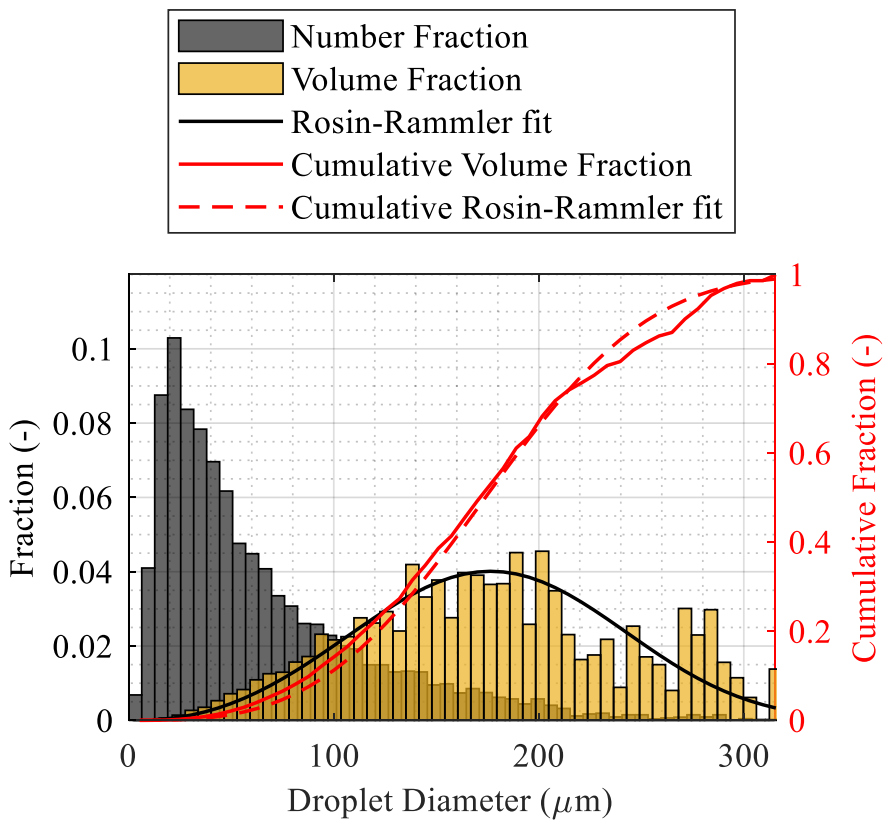

FIGURE 10: ROSIN-RAMMLER FIT ON THE VOLUME FRACTION OF THE DROPLET SIZE DISTRIBUTION AT X $=0 \mathrm{~mm}$ $\operatorname{AND} \varphi=81^{\circ}$.

Fitting the Rosin-Rammler equation on the droplet distribution data for each different timestamp during the period, the two parameters were obtained. In Figure 11, the $\mathrm{D}_{63.2 \%}$ parameter is clearly oscillating in time in a similar way to the SMD of the spray. The spread parameter $q$ also shows a slight oscillation while it is mostly maintained in the range of 2 to 3 . The two vertical dashed lines indicate the time instances for which the two probability density functions in Figure 9 were shown; both selections correspond to points near the maximum and minimum of the Rosin-Rammler distribution parameters. The smaller the q parameter, the more wide the distribution is, while as it approaches infinity the distribution collapses at a single diameter. On the other hand, larger values of the $\mathrm{D}_{63.2 \%}$ parameter shift the distribution to higher diameters. Therefore, it makes sense that the periodic change of the spray distribution is mainly governed by the $\mathrm{D}_{63.2 \%}$ parameter.

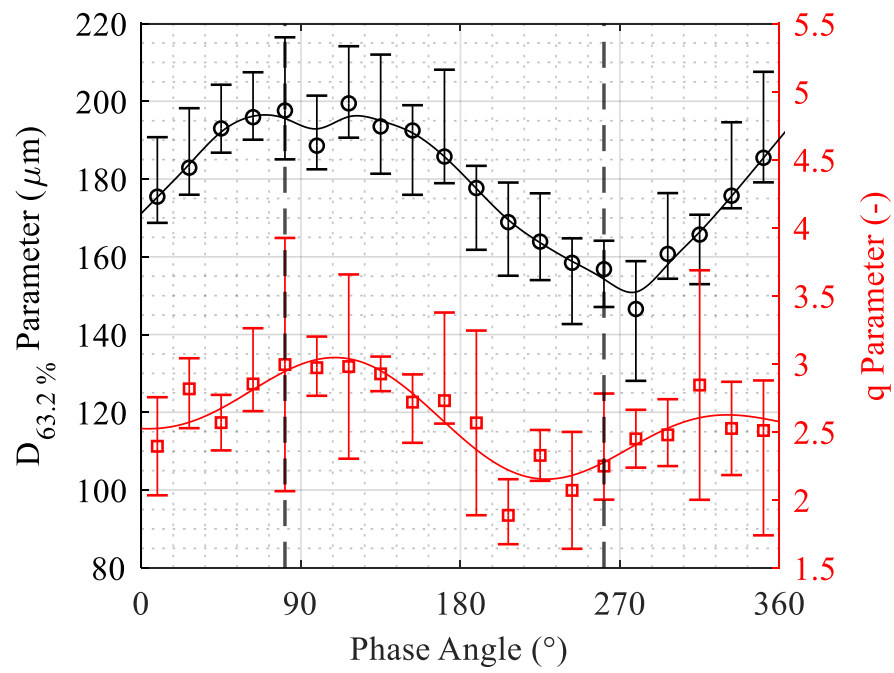

FIGURE 11: PARAMETERS OF ROSIN-RAMMLER DROPLET SIZE DISTRIBUTIONS AT $\mathrm{X}=0 \mathrm{~mm}$.

\section{Derived spray characteristics}

The PDA system measures two velocity components and the diameter for each detected droplet. The aforementioned results illustrated from Figure 4 to Figure 7 are based on the direct measurements from this experimental campaign. By utilizing also the information about the arrival time of each droplet on the PDA measurement volume and the corresponding transit time (the time a droplet needs to cross the volume, in the scale of $\mu \mathrm{s}$ ), the volume and mass flow of the spray per unit area on each position can be calculated. The algorithm proposed by Saffman [14,15] takes into account the mentioned time stamps for each droplet as well as the geometry of the PDA setup in order to estimate the effective area for the mass flux calculation:

$$
\dot{\mathrm{m}}_{\mathrm{L}}^{\prime \prime}=\rho_{\mathrm{L}} \frac{\pi}{6 \Delta \mathrm{t}} \sum_{\mathrm{i}=1}^{\mathrm{N}} \frac{\mathrm{d}_{\mathrm{i}}^{3}}{\mathrm{~A}_{\mathrm{i}}}
$$

where $\rho_{L}$ is the density of the liquid, $d_{i}$ is the diameter of each droplet from a total of $\mathrm{N}$ droplets, $\mathrm{A}_{\mathrm{i}}$ is the effective detection area for each droplet and $\Delta \mathrm{t}$ is the total measurement time. For each measured position in the spray, a single value of the mass flux is usually calculated. To evaluate the calculation of the mass flux, measurements under non-forced conditions were conducted, where the pressure drop at the nozzle was the same as the average pressure drop in the forced case. A variety of positions on the XY plane was selected, at a downstream distance of $Z=40 \mathrm{~mm}$, covering the whole spray cross-sectional area. 
The outcome is illustrated in Figure 12, where the data on the measured positions are represented by the black dots, while a fifth-order polynomial surface fits these data.

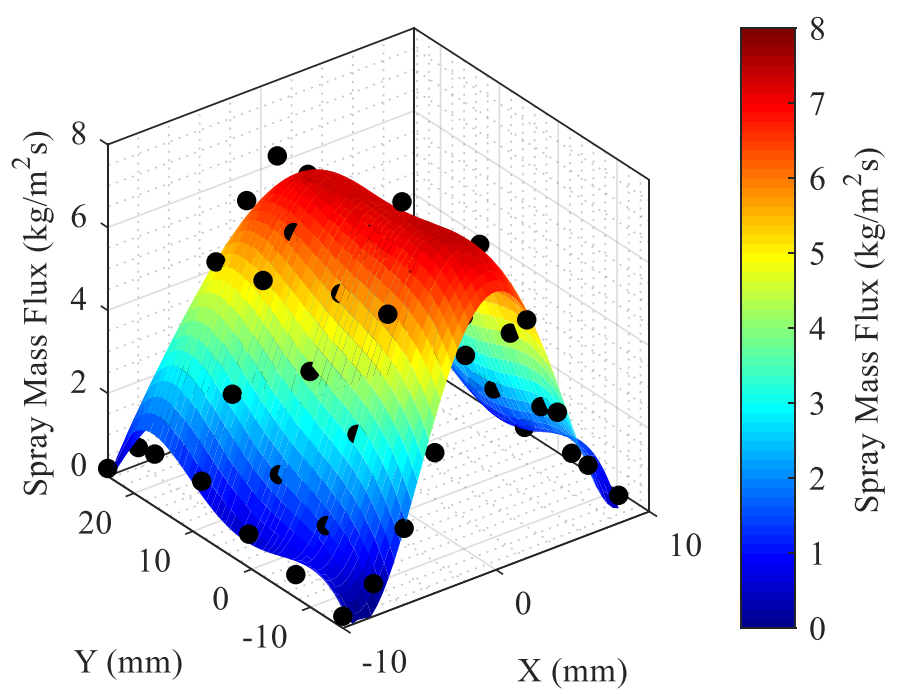

FIGURE 12: MASS FLUX OF SPRAY AT THE $Z=40 \mathrm{~mm}$ PLANE UNDER NON-FORCED CONDITIONS.

The spray mass flux shows a local maximum around $\mathrm{X}=0 \mathrm{~mm}$, indicating that the spray is denser near the centerline and scarce in the radial direction. At the edges of the interval in the $\mathrm{X}$-axis the mass flux reaches near zero values, establishing that the spray region was almost completely covered. The mass flow rate can be estimated by calculating the volume under the surface fit on the mass flux data. The result mass flow rate in $\mathrm{kg} / \mathrm{h}$ was close to the actual mass flow provided by the flow meter, with less than $9 \%$ deviation. Therefore, the calculation of the mass flux via Equation (2) is considered reliable for further application in the forced conditions.

\begin{tabular}{|c|c|c|c|c|c|}
\hline$\Phi$ & $X=-10 \mathrm{~mm}$ & $\bar{\Phi}$ & $X=-6 \mathrm{~mm}$ & $\Phi$ & $X=-2 \mathrm{~mm}$ \\
\hline 巫 & $X=-8 \mathrm{~mm}$ & $\Phi$ & $\mathrm{X}=-4 \mathrm{~mm}$ & 平 & $\mathrm{X}=0 \mathrm{~mm}$ \\
\hline
\end{tabular}

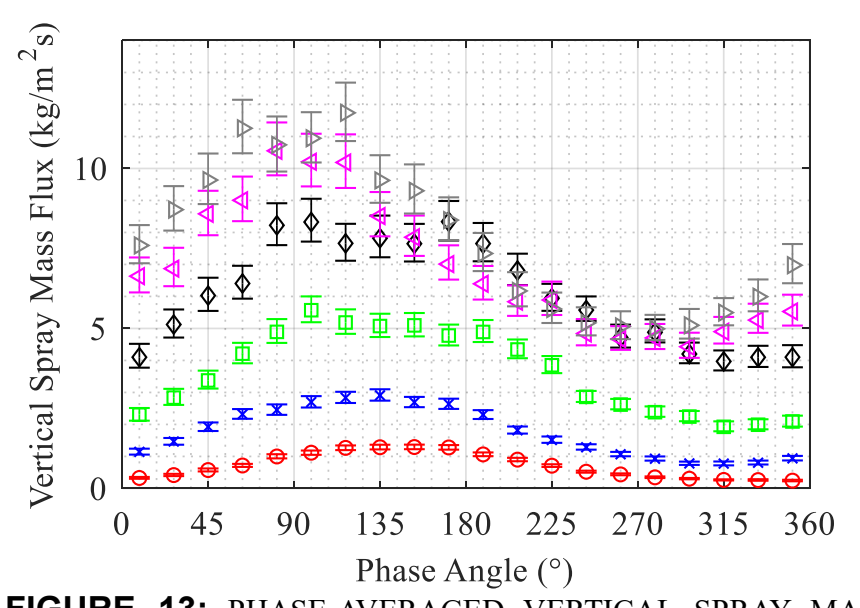

FIGURE 13: PHASE-AVERAGED VERTICAL SPRAY MASS FLUX AT $93.5 \mathrm{~Hz}$.
In the context of this study, since the duration of each forced experiment was phase-averaged in twenty subsections, the mass flux in each position should also oscillate in time. In Figure 13, the phase-averaged vertical mass flux component, which is the vertical (parallel to the Z-axis), is illustrated, where a clear fluctuation in time is observed. As expected due to the observations of Figure 12, moving radially from the centerline ( $\mathrm{X}=0 \mathrm{~mm}$ ) of the spray the vertical mass flux component is steeply decreasing. This indicates that the majority of the droplets are closer to the center of the spray in the forced experiments as well, where there is an average of $7.7 \mathrm{~kg} / \mathrm{m}^{2} \mathrm{~s}$. Even though the mass flux calculation is based on both the velocity and the diameter of the droplet, it is evident that higher values are observed where the SMD of the spray is larger, showing that the size of the droplet is dominant over its velocity concerning the mass flux.

Having established the fact that the spray mass flux oscillates in time due to the acoustic forcing imposed in the airflow, even though the water supply was constant, a question regarding the behavior of the air-to-liquid ratio arises. The ALR is expressed as the mass flow rate of the air over the mass flow rate of the liquid:

$$
\mathrm{ALR}=\frac{\dot{\mathrm{m}}_{\mathrm{A}}}{\dot{\mathrm{m}}_{\mathrm{L}}}=\frac{\rho_{\mathrm{A}} \mathrm{u}_{\mathrm{A}}}{\dot{\mathrm{m}}_{\mathrm{L}}^{\prime \prime}}
$$

In this case, where the mass flux of the liquid is calculated based on the algorithm developed by Saffman, the second expression of Equation (3) can be used. For the air mass flow rate, the phaseaveraged air velocity shown in Figure 3 was utilized. Because both the velocity on the numerator and the mass flux on the denominator are phase-averaged in one period, the same applies to the resulting ALR.

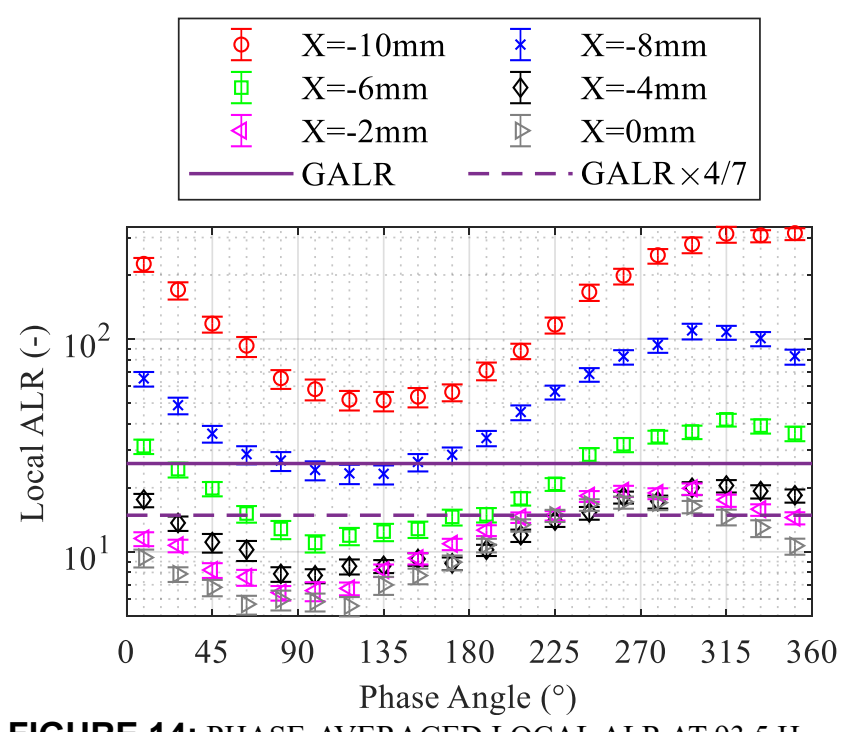

FIGURE 14: PHASE-AVERAGED LOCAL ALR AT 93.5 Hz.

The local ALR calculated in each measurement position is shown in Figure 14. A strong periodic oscillation is observed in 
all different positions, starting from low average values at the center of the spray at $\mathrm{X}=0 \mathrm{~mm}$, ending in values higher than 100 at the edge of the measured interval at $X=-10 \mathrm{~mm}$. This was expected since the spray is dense close to its centerline and, even though it does spread to the sides as well, its mass flux there is radically decreasing (Figure 13). The y-axis in Figure 14 is on a logarithmic scale, in order to better visualize the periodic fluctuation in all six measured locations of the spray.

On the same graph, two straight lines have been highlighted. The solid one refers to the average global ALR (GALR) of the system, which indicates the total average mass flow rate of the air supplied in the system over the total water mass flow rate, which was approximately 26 . The dashed line is simply a specific fraction of the GALR. On the prefilmer shown in Figure 2 , the air is flowing obviously on the whole duct with a width of $70 \mathrm{~mm}$, while the water film covers a width of only $40 \mathrm{~mm}$, due to the positioning of the holes. Therefore, a $4 / 7$ fraction of the GALR also makes sense in the context of comparing with the calculated local ALR. These two GALR values coincide with the oscillating local ALR values observed near the positions $-6 \mathrm{~mm}$ to $-4 \mathrm{~mm}$.

\section{Comparison to non-forced \& amplitude analysis}

Finally, it is useful to observe the basic spray characteristics under non-forced conditions, in operation where the pressure drop at the nozzle was the same as the average pressure drop in the forced experiments. The mean vertical droplet velocity is juxtaposed with the SMD of the spray in Figure 15, for the same measurement positions shown in Figure 2

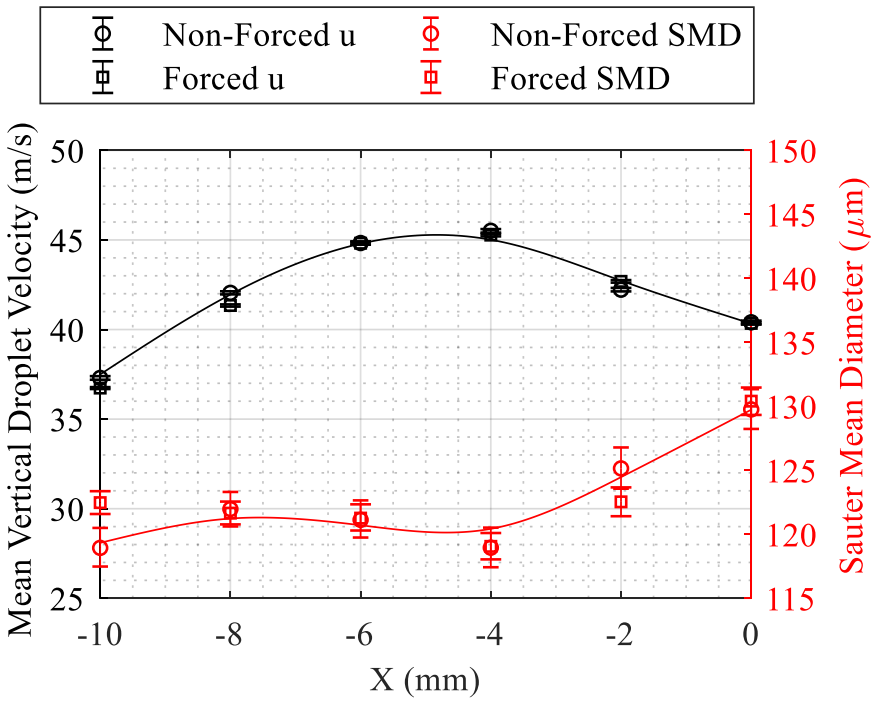

FIGURE 15: MEAN DROPLET VELOCITY AND SMD ALONG THE X-AXIS FOR FORCED AND NON-FORCED CONDITIONS.

The mean velocity seems to increase moving from the centerline to the extreme for the first 4 to $6 \mathrm{~mm}$, after which it drops again. On the other hand, the SMD of the spray shows a local maximum around $\mathrm{X}=0 \mathrm{~mm}$ and then remains rather constant. As already observed from Figure 4 and Figure 7, the same behavior was found on the forced case spray characteristics. For this reason, the average velocity of the spray and the SMD of the spray on each position for the forced flow experiments are shown in Figure 15 as well, treated as if they behaved quasi-steady. It is evident that there is no significant difference between the non-forced spray characteristics and their counterparts in experiments with an oscillation in the airflow.

Since the behavior match between the two cases was established, it is interesting to quantify how strong the fluctuation of each of the spray characteristics is. By calculating the amplitude of the oscillation for the velocities, the angle, the SMD, the mass flux, and the ALR, on each of the six measurement positions, it is possible to examine and compare how influenced they were due to the air forcing. For the mean vertical droplet velocity and the SMD, the average of the forced case has already been shown to match the non-forced values in Figure 15, while the same applies for all other spray characteristics. Therefore, the amplitude of each parameter was calculated as a percentage relative to their average value.

\begin{tabular}{|clcl|}
\hline$\Phi$ & Vertical Velocity & $\Phi$ & SMD \\
$\bar{\Phi}$ & Horizontal Velocity & $\Phi$ & Spray Mass Flux \\
$\bar{\Phi}$ & Angle & $\Phi$ & Local ALR \\
\hline
\end{tabular}

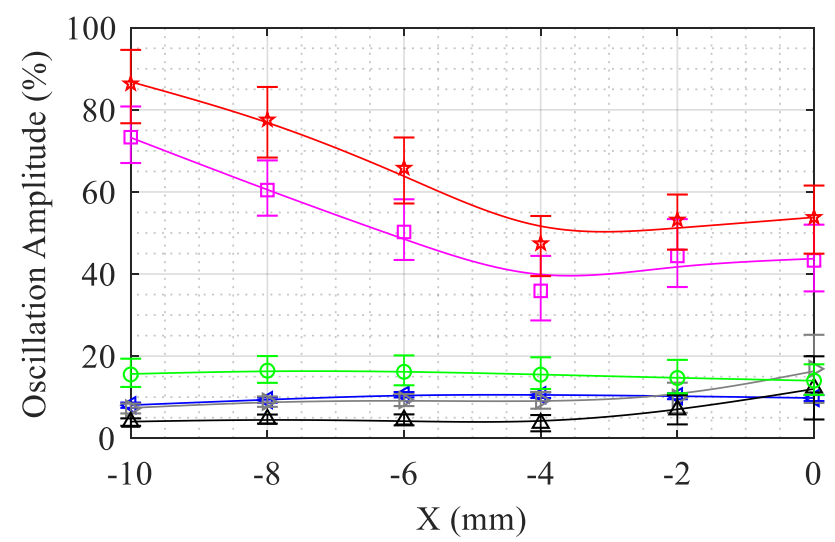

FIGURE 16: RELATIVE AMPLITUDES OF PHASE-AVERAGED MAIN SPRAY CHARACTERISTICS AT $93.5 \mathrm{~Hz}$.

In Figure 16, the velocity angle has the lowest relative amplitude since it was already established that it remains almost constant in time. The two velocity components are also quite low, with an amplitude near $10 \%$ of their average, while for the SMD it is also between $10 \%$ and $20 \%$ due to its high average value. The spray mass flux has a significantly higher relative oscillation amplitude compared to the other spray characteristics. As a result, the local ALR also oscillates with a high amplitude, starting from $55 \%$ at $\mathrm{X}=0 \mathrm{~mm}$ reaching even $85 \%$ at $\mathrm{X}=$ $10 \mathrm{~mm}$. Such a strong oscillation in the local ALR would definitely influence the flame stability and potentially risk the engine hardware.

\section{CONCLUSION}

The influence of an acoustically excited airflow on the prefilming airblast atomization process on a generic planar prefilmer was experimentally investigated. In the setup, a siren 
was generating a pulsating airflow, investigated by means of hot wire Constant Temperature Anemometry, and the generated spray of droplets was characterized via a Phase Doppler Anemometry system. The measured air velocity and droplet characteristics were phase-averaged during the post-processing phase in order to observe their periodic fluctuation. Both the CTA and the PDA systems were mounted on a translation stage, enabling spatial measurements of the airflow and the spray properties, respectively. The benefit of the spatially resolved measurements provided a concrete base for the observed outcomes.

The air velocity was initially characterized, showing a clear periodic behavior, arising the question of how the droplet characteristics would respond to this forcing. The droplet velocity in two components and the droplet diameter were directly measured by the PDA system. Therefore, the mean droplet velocity, as well as the SMD of the spray, could be calculated, and their oscillation during the period was observed. The trend of both the velocity and the SMD along the X-axis matches the corresponding trend under non-forced conditions, highlighting this way the influence that the acoustic forcing adds to the system.

The spray produced has an inverse correlation between the velocity and the diameter, so the SMD of the spray is minimum at the instant when the average droplet velocity is at the maximum of its oscillation. Since the spray spreads along the $\mathrm{X}$ axis, its horizontal velocity component increases in absolute value near the extremes of the investigated interval. In all positions though, the velocity angle seems to remain almost constant with no fluctuation in time. The droplet size distribution also changes periodically, matching the oscillation of the SMD; by applying the Rosin-Rammler model in the different subsections of the period, a periodic fluctuation in the model's parameters is also apparent.

Apart from the data acquired directly from the PDA measurement technique, the mass flux of the spray on each location was also calculated. The vertical mass flux (parallel to the $\mathrm{Z}$-axis) radically decreases moving along the $\mathrm{X}$-axis, showing that the spray is denser in the center. A strong periodic oscillation is apparent for all measured positions, indicating that the rate at which the droplets arrive in a specific location is unsteady, even though the water was supplied at a constant rate in the prefilmer. The calculation of the mass flux was verified in non-forced experiments on the XY plane, through which the mass flow rate of the system was reconstructed.

Using the calculated mass flux and the air velocity measured via hot wire anemometry, the calculation of a local air-to-liquid ratio was possible. The ALR similarly undergoes a strong periodic fluctuation and its average increases moving along the $\mathrm{X}$-axis. The global ALR lies inside the investigated interval of $10 \mathrm{~mm}$ from the centerline and fluctuates around the middle of that distance.

Finally, in order to quantify the periodic fluctuations of all investigated spray parameters, their amplitude was calculated. The average of the oscillating spray characteristics was close to the corresponding value of the non-forced experiments, therefore the amplitudes were calculated as a percentage relative to their average value. The angle of the spray has a negligible amplitude while the velocities stay below $20 \%$ of their respective average. The relative oscillation amplitude of the SMD stays also near $20 \%$, which is not insignificant since it corresponds to approximately $40 \mu \mathrm{m}$. Based on the strong oscillation of the SMD, the mass flux and the local ALR undergo a wide periodic fluctuation with relative amplitudes in the range of $40 \%$ to $85 \%$, indicating possible combustion stability and engine safety issues, highlighting the demand to predict the instabilities on the spray before they occur.

\section{ACKNOWLEDGEMENTS}

This project has received funding from the European Union's Horizon 2020 research and innovation programme under the Marie Sklodowska-Curie Agreement 766264 (MAGISTER). The authors would like to thank the Institute of Thermal Turbomachinery (ITS) in KIT for the expertise regarding the planar prefilming airblast atomizer model, as well as the Chair of Thermodynamics in TUM for manufacturing, testing, and providing the pulsation device. Finally, the authors would like to gratefully acknowledge the financial support by the German Research Foundation (DFG) for measuring equipment under HBFG programme INST 121384/178-1 FUGG.

\section{REFERENCES}

[1] Gokulakrishnan P., Ramotowski M. J., Gaines G., Fuller C., Joklik R., Eskin L. D., Klassen M. S., and Roby R. J., 2008, "A novel low NOx lean, premixed, and prevaporized combustion system for liquid fuels," Journal of Engineering for Gas Turbines and Power, 130(5), pp. 1-7. doi:10.1115/1.2904889.

[2] DeLaat J. C., and Paxson D. E., "Characterization and simulation of the thermoacoustic instability behavior of an advanced, low emissions combustor prototype. 44th AIAA/ASME/SAE/ASEE Joint Propulsion Conference \& Exhibit, Hartford, CT, USA, July 21-23, 2008. doi:10.2514/6.2008-4878.

[3] Lefebvre A. H., and Ballal D. R., Gas Turbine Combustion: Alternative Fuels and Emissions, CRC Press Taylor \& Francis Group, LLC, Boca Raton (2010).

[4] Lieuwen T. C., and Yang V., Combustion Instabilities in Gas Turbine Engines: Operational Experience, Fundamental Mechanisms, and Modeling, American Institute of Aeronautics and Astronautics, Inc., Reston (2005).

[5] Eckstein J., Freitag E., Hirsch C., Sattelmayer T., Bank R. von der, and Schilling T., 2005, "Forced Low-Frequency Spray Characteristics of a Generic Airblast Swirl Diffusion Burner," Journal of Engineering for Gas Turbines and Power, 127(2), pp. 301-306. doi:10.1115/1.1789515.

[6] Müller A., Koch R., Bauer H.-J., Hehle M., and Schafer O., "Performance of Prefilming Airblast Atomizers in Unsteady Flow Conditions. Proceedings of ASME Turbo 
Expo 2006, Barcelona, Spain, May 8-11, 2006. doi:10.1115/GT2006-90432.

[7] Chaussonnet G., Müller A., Holz S., Koch R., and Bauer H.-J., 2017, "Time-Response of Recent Prefilming Airblast Atomization Models in an Oscillating Air Flow Field," Journal of Engineering for Gas Turbines and Power, 139(12), 121501 1-9. doi:10.1115/1.4037325.

[8] Su J., Barker A., Garmory A., and Carrotte J., "Spray Response to Acoustic Forcing of a Multi-Passage LeanBurn Aero-Engine Fuel Injector. Proceedings of ASME Turbo Expo 2018, Oslo, Norway, June 11-15, 2018. doi:10.1115/GT2018-75554.

[9] Efron B., and Tibshirani R. J., An Introduction to the Bootstrap, Springer Science+Business Media, Dordrecht (1993).

[10] Montgomery D. C., and Runger G. C., Applied statistics and probability for engineers, 3rd ed., Wiley, New York (2003).

[11] Collins L. R., and Keswani A., 2004, "Reynolds number scaling of particle clustering in turbulent aerosols," New Journal of Physics, 6(119), 1-17. doi:10.1088/13672630/6/1/119.

[12] Mugele R. A., and Evans H. D., 1951, "Droplet Size Distribution in Sprays," Industrial \& Engineering Chemistry, 43(6), pp. 1317-1324. doi:10.1021/ie50498a023.

[13] Lefebvre A. H., and Mcdonell V. G., Atomization and Sprays, CRC Press Taylor \& Francis Group, LLC, Boca Raton (2017).

[14] Saffman M., 1987, "Automatic calibration of LDA measurement volume size," Applied Optics, 26(13), pp. 2592-2597. doi:10.1364/ao.26.002592.

[15] Qiu H.-H., and Sommerfeld M., 1992, “A reliable method for determining the measurement volume size and particle mass fluxes using phase-Doppler anemometry," Experiments in Fluids, 13(6), pp. 393-404. doi:10.1007/bf00223247. 